\title{
Etude expérimentale des voies d'élimination de l'azote en présence de trois microalgues - Synechocystis, Chlorella, Micractinium - : évaluation de l'assimilation algale, de la nitrification et du stripping de l'ammoniac
}

\author{
L. Bouarab 1 \\ M. Ould El Mehdi ${ }^{1}$ \\ B. Sbiyyaa ${ }^{1}$ \\ A. Ait Melloul ${ }^{2}$ \\ B. Oudra 1 \\ M. Loudiki ${ }^{1}$ \\ L.Hassani2 \\ A. Dauta ${ }^{3}$
}

Mots-clés : azote, nitrification, stripping, bactérie, eaux usées, lagunage, algue, Synechocystis, Chlorella, Micractinium.

Trois microalgues, Synechocystis (Picocyanobactérie), Chlorella et Micractinium (Chlorophyta) se développant dans le lagunage naturel, ont été mises en culture en milieu axénique et ensemencées dans des microcosmes d'eau usée filtrée, stérilisée ou non. Ceci dans le but d'évaluer les voies d'élimination de $\mathrm{NH}_{4}$, apporté par cette eau usée. L'assimilation algale, la nitrification et le stripping de $\mathrm{NH}_{3}$, se sont révélées les principales voies d'élimination de l'ammonium dans le milieu non stérilisé. Par ailleurs, dans le milieu stérilisé, seuls l'assimilation algale et le stripping sont présents. Les bactéries hétérotrophes aérobies semblent ne pas intervenir dans cette élimination.

Experimental study of nitrogen removal in the presence of three algal species - Synechocystis, Chlorella, Micractinium - : evaluation of algal uptake rate, nitrification and ammonia stripping process

Keywords : nitrogen, nitrification, stripping, bacteria, wastewater, stabilization pond, algae, Synechocystis, Chlorella, Micractinium.

Three microalgae species : Synechocystis (Picocyanobacteria), Chlorella and Micractinium (Chlorophyta) isolated from wastewater stabilisation ponds, were cultured on a mineral medium. In order to evaluate the removal rate depending on different processes and microalgae culture conditions, algal growth was studied both on sterilized and non sterilized sewage water filtrate. The results show that algal uptake, nitrification and $\mathrm{NH}_{3}$ stripping are the principal process leading to $\mathrm{NH}_{4}$ removal under nonsterilized conditions. However, the nitrification process do not occur on sterilized microcosms. It seems that aerobic heterotrophic bacteria did not act on the $\mathrm{NH}_{4}$ removal process. According to each process, culture condition and utilized microalgae strain, the evaluation of various nitrogenous removal rates are discussed.

\section{Introduction}

La pollution de l'environnement par l'azote inorganique est le résultat d'un déséquilibre entre les apports de l'azote sous forme de l'ammonium et ses transfor-

1. Laboratoire d'Algologie, Université Cadi Ayyad, Faculté Sciences Semlalia, B.P. S/ 15, Marrakech, 40.000, Maroc.

2. Laboratoire de Microbiologie, Université Cadi Ayyad, Faculté Sciences Semlalia, B.P. S/ 15, Marrakech 40 000, Maroc.

3. Centre d'Écologie des Systèmes Aquatiques Continentaux UMR C 5576 CNRS-Université Paul Sabatier, 118 route de Narbonne, F31062 Toulouse Cedex 04, France. mations sous forme de $\mathrm{N}_{2}$; cette dernière forme est considérée sans danger, alors que toutes les autres formes peuvent créer, en situation de déséquilibre, des nuisances écologiques (Abeliovich,1992). Les formes inorganiques, $\mathrm{NH}_{4}, \mathrm{NO}_{2}$ et $\mathrm{NO}_{3}$ peuvent déclencher, non seulement le phénomène d'eutrophisation des écosystèmes aquatiques, mais sont à des degrés variables toxiques pour différentes formes de vie. Le traitement par différents systèmes des eaux chargées en azote telles les eaux usées, source de ces problèmes, a fait l'objet de nombreux travaux de par le monde. Dans le lagunage naturel, l'azote est éliminé par plusieurs mécanismes : 1) assimilation biologique de $\mathrm{NH}_{4}, 2$ ) Strip- 
ping de $\mathrm{NH}_{3}$, 3) Nitrification-Dénitrification, 4) Sédimentation de l'azote organique (Middlebrooks et al. 1983). L'assimilation biologique est généralement affectée par la température, la charge organique, le temps de séjour et les caractéristiques des eaux usées. L'assimilation du phytoplancton dépend de l'état physiologique des cellules, des quotas intracellulaires, du rapport biosurface/ biovolume et du rapport N/P (Rhee 1978 ). Il est généralement admis que la valeur de ce dernier rapport se situe dans un milieu équilibré autour de 15 (Redfield ratio). En présence des formes inorganiques de l'azote, l'assimilation est sous la dépendance de l'affinité (Ks) des microalgues vis-à-vis de ces formes. En effet, $\mathrm{NH}_{4}$ est préférentiellement assimilé vis-à-vis des nitrates; certains auteurs, cependant (Robert \& Maestrini 1986) rapportent qu'il y a également assimilation de $\mathrm{NO}_{3}$ mais à un faible taux. Ceci est expliqué par une inhibition de la nitrate réductase en présence de $\mathrm{NH}_{4}$ (Syrett 1981). L'ammonium et les nitrites, à des concentrations élevées, sont toxiques pour les microalgues en ce sens qu'ils affectent leur activité photosynthétique.

L'assimilation des bactéries hétérotrophes dépend de l'azote organique dissous et pour une large fraction de $\mathrm{NH}_{4}$. L'assimilation de $\mathrm{NO}_{3}$ et de l'urée est relativement faible. Dans une étude sur le milieu marin, Krichman et al. (1994) rapportent que la production bactérienne suit celle du phytoplancton en ce sens que les bactéries se développent surtout à partir des acides aminés de faible poids moléculaire (Dissolved free amino acids «D.F.A.A.»). Coveney et al. (1977) expliquent cette succession algues - bactéries dans un lac eutrophe par l'autolyse et l'excrétion de produits organiques par le phytoplancton. Watt cité par Coveney et al. (1977) a montré qu'en présence de Stephanodiscus, il y a relargage prédominant du glycolate, acide aminé qui est rapidement assimilé par les bactéries.

Le taux des pertes par stripping dépend surtout du $\mathrm{pH}$, de la température, des conditions de mélange et de la charge hydraulique.

La nitrification est réalisée en milieu aérobie et en présence des populations bactériennes nitrifiantes, essentiellement Nitrosomonas et Nitrobacter. L'activité nitrifiante dépend des conditions environnementales température, $\mathrm{pH}$, oxygène dissous, temps de séjour - et des caractéristiques physico-chimiques des eaux usées.

Les données bibliographiques donnent des conclusions très controversées quant à l'importance de ces processus dans le lagunage naturel (Santos \& Oliveira 1987, Somiya \& Fujii 1984, Ferrara \& Avci 1982, Ellis 1983, Neel et al. 1961, Mujeriego et al. 1993, Walker \& Leclerc 1973, Middlebrooks \& Pano 1983). L'évaluation in situ de ces processus étant très difficile, nous nous sommes orientés vers une expérimentation en laboratoire en culture discontinue. Ce type d'expérience est facile à mettre en œuvre, moins coûteux , mais il est critiqué par sa faible représentation de la réalité de l'écosystème. Le but de ce travail est l'étude de quelques mécanismes mentionnés ci-dessus ainsi que la capacité épuratoire de trois espèces d'algues dans des milieux confectionnés avec des eaux usées stériles ou non. Les espèces d'algues utilisées sont une Picocyanobactérie (Synechocystis) et deux Chlorophycées (Chlorella et Micractinium).

\section{Matériel et méthodes}

\subsection{Milieux de culture}

L'effluent est celui de la station expérimentale de Marrakech. Le Tableau 1 donne les caractéristiques de ces eaux usées typiquement domestiques. Au laboratoire, l'eau est filtrée sur filtre Whatman GF/C $(1,2$ $\mu \mathrm{m})$ qui est suffisant pour supprimer les matières en suspension, y compris le phytoplancton composé, pendant la période de collecte, principalement de Chlamydomonas sp. Les milieux synthétiques utilisés pour le maintien des algues sont les suivants : milieu DAUTA pour Micractinium, milieu BG13 pour Synechocystis et le milieu BOLD pour Chlorella.

Tableau 1. Caractéristiques physico-chimiques des eaux usées utilisées dans les expérimentations.

Table 1. Physico- chemical characteristics of wastewater effluent used in the experiments.

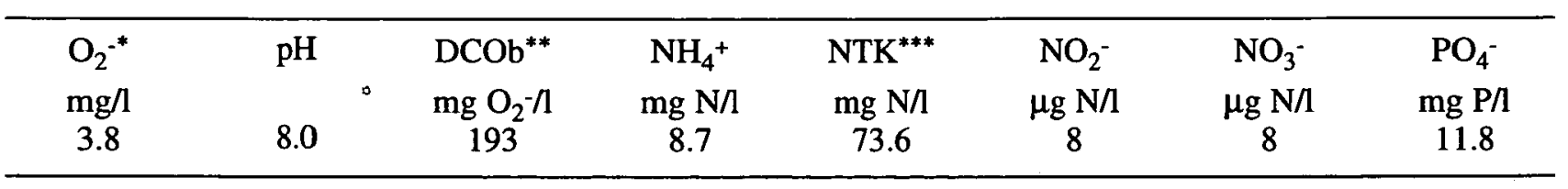

* oxygène dissous ; ** DCO brute ; ${ }^{* *}$ Azote total Kjeldahl. 


\subsection{Mode de culture}

Deux types de milieux de culture en batch (volume : 1,61 ) d'eau usée soit stérilisée par passage à l'autoclave $\left(15 \mathrm{~min}\right.$. à $\left.120^{\circ} \mathrm{C}\right)$ soit non stérilisée, sont confectionnés dans des erlenmeyers de deux litres et inoculés chacun (en duplicata) par l'une des trois algues étudiées. La stérilisation permet d'éliminer les bactéries apportées par les eaux usées. Avec l'inoculation par les différentes espèces d'algues, l'évolution de $\mathrm{NH}_{4}$ est uniquement sous leur effet sur milieu stérilisé ; alors qu'elle est sous l'effet combiné des bactéries et des algues sur milieu non stérilisé. Les expériences se déroulent dans une salle thermostatée à $26^{\circ} \mathrm{C}$ sous un éclairage continu de $62 \mu \mathrm{E} \cdot \mathrm{m}^{-2} \cdot \mathrm{s}^{-1}$. L'agitation du milieu est assurée par bullage. Les milieux de culture à base d'eau usée sont ensemencés avec des cellules algales en phase exponentielle de croissance et en parfait état nutritionnel. Comme les milieux synthétiques sont très riches en substrat azoté $\left(\mathrm{NO}_{3} \mathrm{~K}\right)$, les stocks intracellulaires sont à leur quota maximum. Ceci évite de travailler avec des cellules carencées qui réagissent par une forte prise en charge de l'élément nutritif déficient (Caperon \& Meyer 1972, Dauta 1982, Lavoie \& De la Noue 1985). En plus de ces expériences, nous avons procédé à un enrichissement en $\mathrm{NH}_{4} \mathrm{Cl}$ à partir du 5 ème jour, sur la deuxième série du milieu de culture en présence de Chlorella afin de maintenir la concentration des ions ammonium au même niveau.

\subsection{Méthodes d'analyse}

Les différentes formes de l'azote minéral sont dosées dans l'eau filtrée. L'ammonium est dosé par la méthode colorimétrique au bleu d'indophénol (Norme AFNOR T90-015 ) ; le stripping de $\mathrm{NH}_{3}$ est estimé par piégeage de $\mathrm{NH}_{3}$ dans une solution d'acide borique $(50 \mathrm{ml}$ à $0,32 \mathrm{M}$ ) puis dosé par la méthode au bleu d'indophenol ; les nitrites sont dosés par méthode colorimétrique reposant sur la diazotation par la sulfanilamide (Norme AFNOR T 90-013) ; les nitrates sont dosés par colorimétrie des nitrites après réduction sur colonne Cadmium Cuivre. L'azote Kjeldhal (NTK) est déterminé après minéralisation de l'azote organique en ammonium en milieu acide et en présence de catalyseur à base de sélénium (Norme AFNOR T90 110).

La biomasse des algues est estimée par dosage de la chlorophylle a, en utilisant de l'ethanol porté à ébullition pour l'extraction (Marker et al. 1980). La densité cellulaire évaluée par numération cellulaire à l'aide d'un hematimètre Malassez, est exprimée en nombre de cellules par litre.

L'abondance des bactéries hétérotrophes aérobies est estimée par le dénombrement des unités formant colonies (U.F.C) sur gélose nutritive incubée à $30^{\circ} \mathrm{C}$ en duplicata pendant une semaine.

\subsection{Méthodes de calculs}

La quantité d'azote consommée par les algues dans le milieu non stérile est déterminée selon Wrigley \& Toerien (1990) en supposant que la biomasse algale contient $3 \%$ d'azote. La biomasse, exprimée en matière totale en suspension, est déduite à partir de la chlorophylle a qui forme, selon Wrigley \& Toerien (1990), $0.87 \%$ de cette biomasse. La quantité d'ammonium nitrifié est évaluée par la somme des concentrations maximales en $\mathrm{NO} 2$ et en NO3.

\section{Résultats}

\subsection{Evolution des formes azotées}

Pour les trois espèces d'algues le comportement de l'ion ammonium varie peu dans les milieux stérile et non stérile. L'épuisement de $\mathrm{NH}_{4}$ est un peu plus rapide dans le milieu stérile. Les teneurs qui avoisinent $9 \mathrm{mg} \mathrm{N}-\mathrm{NH}_{4} / \mathrm{L}$ à l'instant $\mathrm{T}=0$ deviennent nulles à partir du 4 ème -5 ème jour pour le milieu stérile et vers le 6 ème jour pour le milieu non stérile (Fig. 1). Ainsi en 6 jours, il y a disparition totale de l'ammonium dans le milieu réactionnel. Pendant les premiers jours, le pourcentage de prise des $\mathrm{NH}_{4}$ varie de 17 à $38 \%$ dans le milieu stérile et varie de 21 à $26 \%$ dans le milieu non stérile. Il faut noter que la chute spectaculaire des $\mathrm{NH}_{4}$ se situe entre le 4 ème et le 6 ème jour; elle s'effectue en un ou deux jours. La forme $\mathrm{NH}_{3}$ apparaît à partir du 4 ème jour, mais elle n'excède pas une teneur maximale de $0.3 \mathrm{mg} \mathrm{N}-\mathrm{NH}_{3} / \mathrm{L}$ ( Fig. 2).

La production des nitrates et des nitrites est absente dans le milieu stérile où aucune activité nitrifiante n'a été détectée (Fig. 3 ) ; les très faibles teneurs enregistrées durant les premiers jours sont présentes au départ dans le milieu réactionnel, les milieux de maintien des algues étant formés à base de sel de nitrates ; les concentrations des nitrites sont comparables aux valeurs dosées dans les milieux non stériles. Par ailleurs, dans ce dernier, les teneurs en formes oxydées de l'azote augmentent à partir du 4 ème_5ème jour, coïncidant ainsi avec la chute des $\mathrm{NH}_{4}$ (Fig. 4 ). Les concentrations en $\mathrm{NO}_{2}$ sont relativement plus élevées que celles des $\mathrm{NO}_{3}$ quelle que soit l'espèce algale. Les valeurs maximales en $\mathrm{NO}_{2}$ et en $\mathrm{NO}_{3}$ sont observées en présence de Micractinium et de Chlorella. Afin de tester l'action d'un enrichissement en $\mathrm{NH}_{4}$ sur la croissance des algues et sur la nitrification, nous avons procédé à un ajout de $\mathrm{NH}_{4} \mathrm{Cl}$ au 5 ème jour dans les flacons ensemencés avec Chlorella. Ceci a permis de constater 

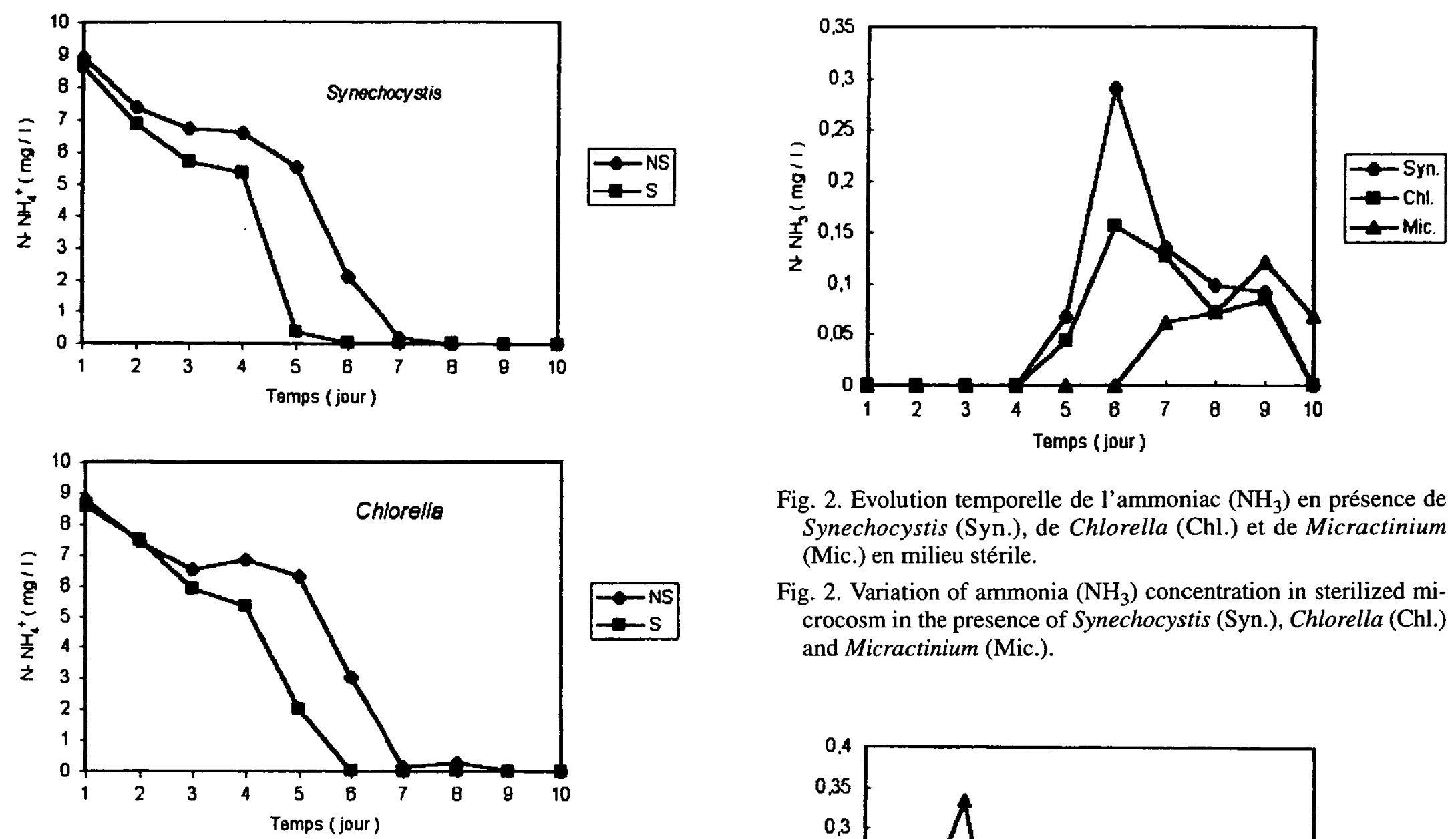

Fig. 2. Evolution temporelle de l'ammoniac $\left(\mathrm{NH}_{3}\right)$ en présence de Synechocystis (Syn.), de Chlorella (Chl.) et de Micractinium (Mic.) en milieu stérile.

Fig. 2. Variation of ammonia $\left(\mathrm{NH}_{3}\right)$ concentration in sterilized microcosm in the presence of Synechocystis (Syn.), Chlorella (Chl.) and Micractinium (Mic.).
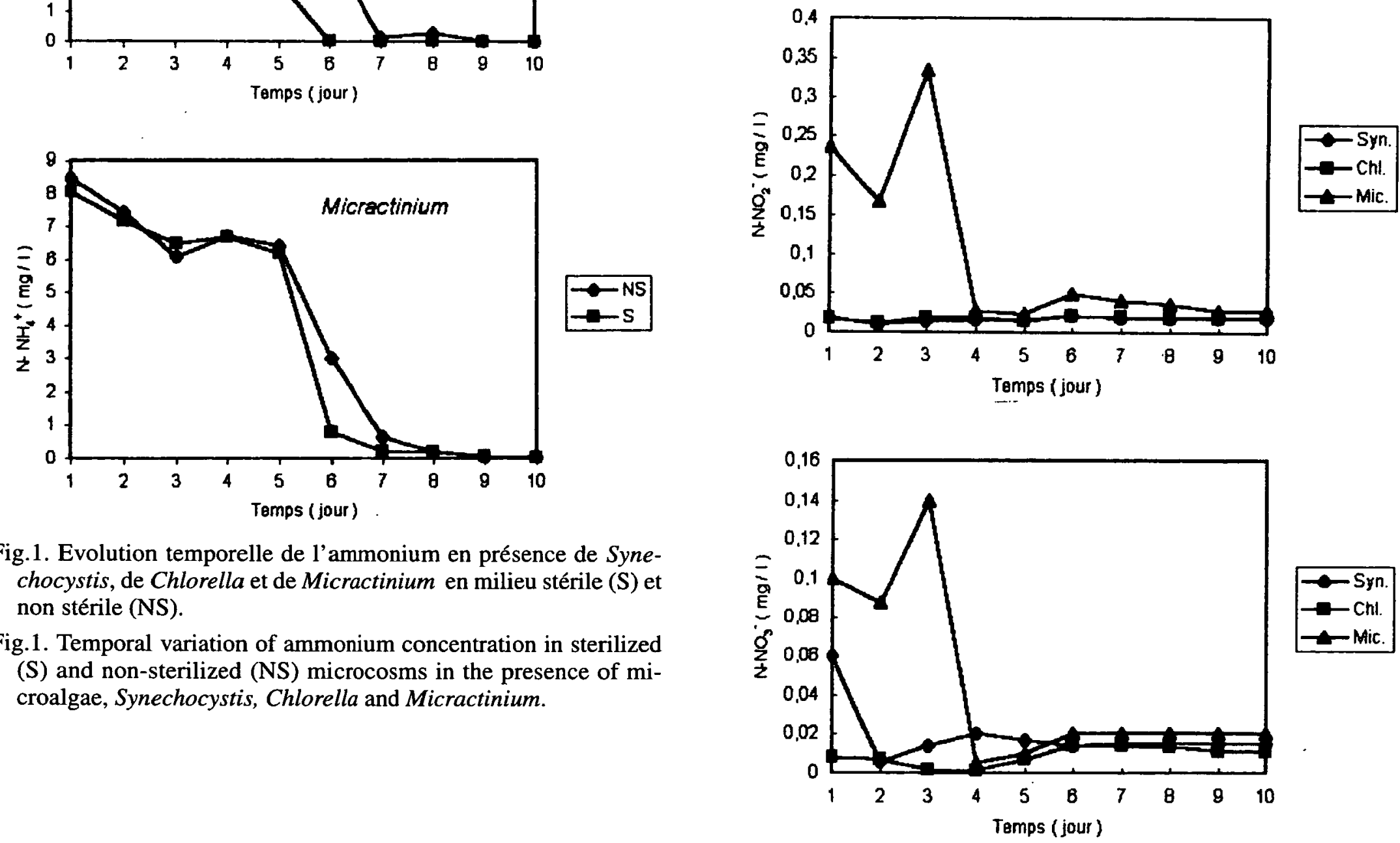

Fig. 3. Evolution des nitrites et des nitrates en présence de Synechocystis (Syn.), de Chlorella (Chl.) et de Micractinium (Mic.) en milieu stérile.

Fig. 3. Variation of nitrite and nitrate concentrations in sterilized microcosms in the presence of Synechocystis ( Syn.), Chlorella (Chl.) and Micractinium (Mic.). 

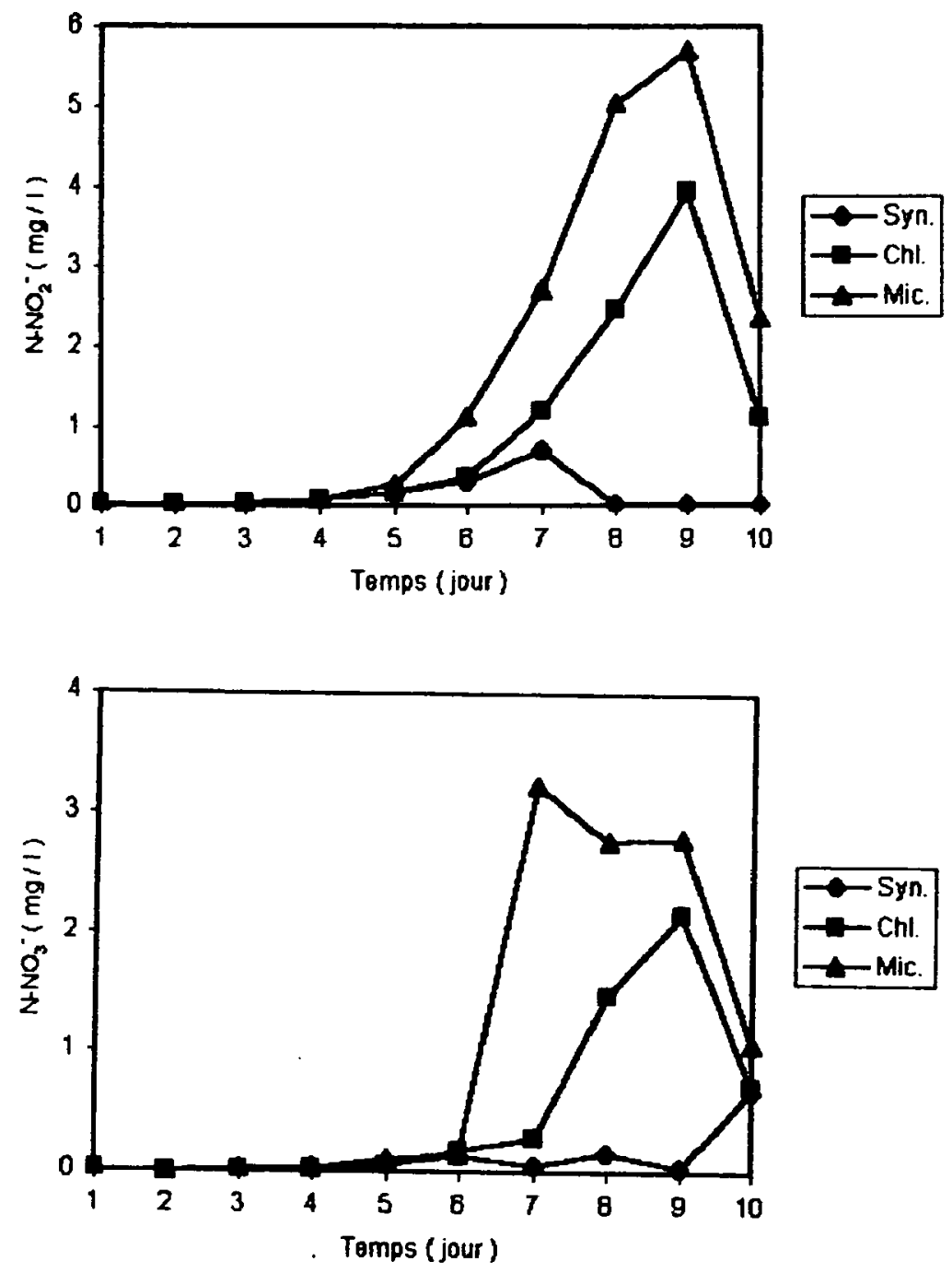

Fig. 4. Evolution des nitrites et des nitrates en présence de Synechocystis (Syn.), de Chlorella (Chl.) et de Micractinium (Mic.) en milieu non stérile.

Fig. 4. Variation of nitrite and nitrate concentrations in non-sterilized microcosms in the presence of Synechocystis (Syn.), Chlorella (Chl.) and Micractinium (Mic.).

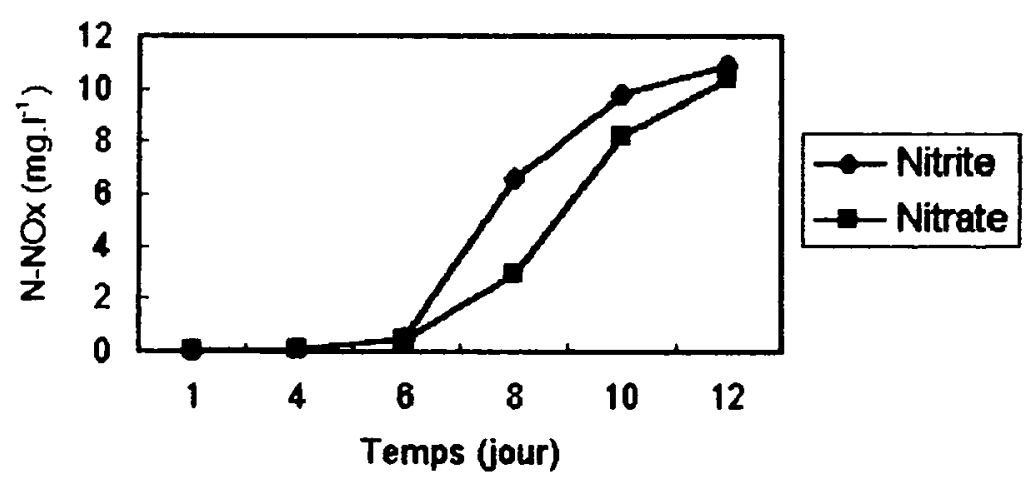

Fig. 6. Evolution des nitrites et des nitrates en présence de Synechocystis en milieu non stérile enrichi en $\mathrm{NH}_{3} \mathrm{Cl}$.

Fig. 6. Nitrite and nitrate concentrations in the presence of Synechocystis in non-sterilized microcosm with ammonium enrichissement using $\mathrm{NH}_{3} \mathrm{Cl}$..
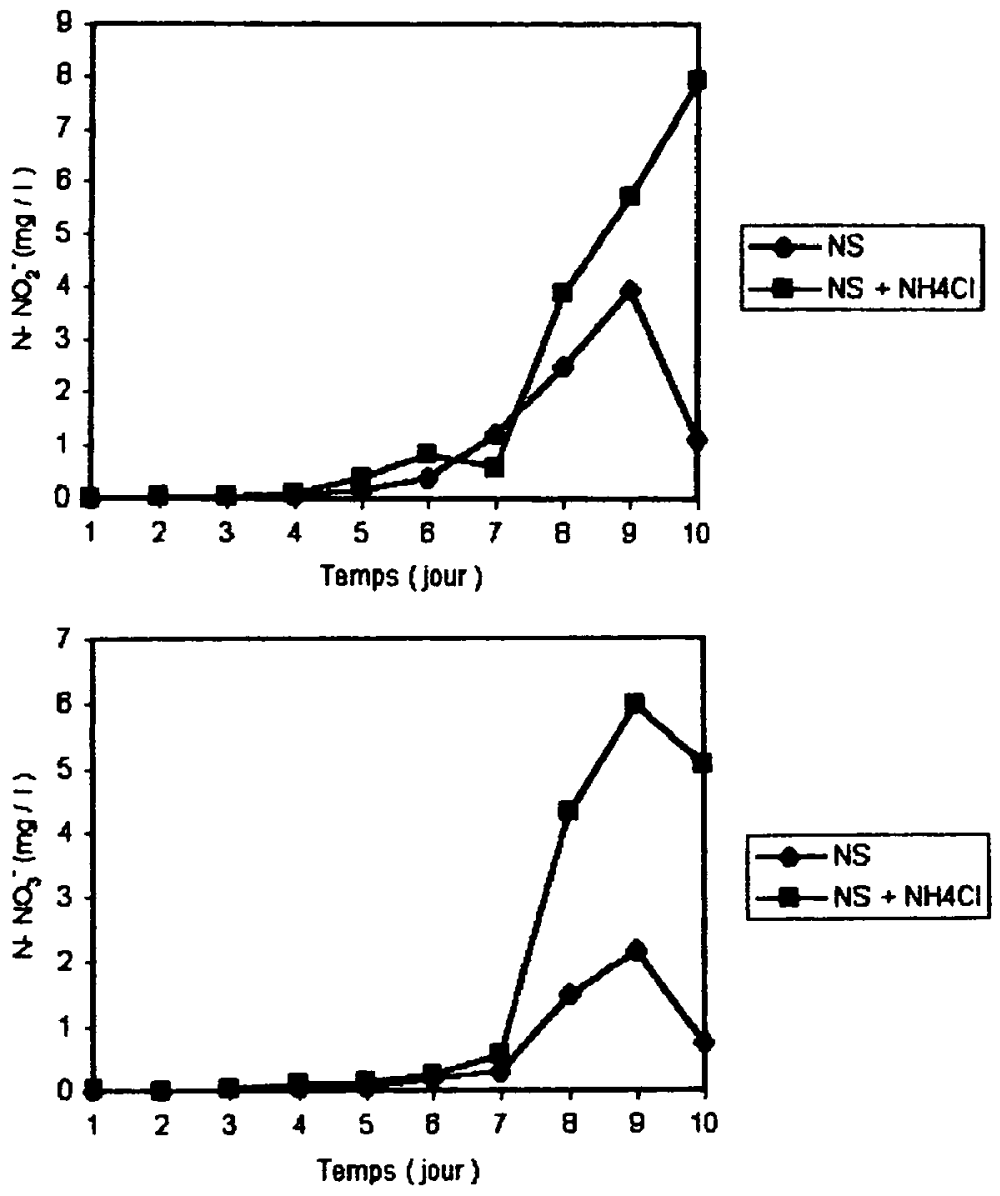

Fig. 5. Evolution des nitrites et des nitrates en présence de Chlorella en milieu non stérile (NS) et non stérile enrichi par $\mathrm{NH}_{4} \mathrm{CL}$ $\left(\mathrm{NS}+\mathrm{NH}_{4} \mathrm{CL}\right.$ ).

Fig. 5. Nitrite and nitrate concentration in presence of Chlorella in non-sterilized microcosm with or without ammonium enrichment using $\mathrm{NH}_{4} \mathrm{Cl}$.

une élévation de l'activité nitrifiante d'environ $50 \%$ pour les $\mathrm{NO}_{2}$ et de $64 \%$ pour les $\mathrm{NO}_{3}$ (Fig. 5 ).

L'absence de nitrification en présence de Synechocystis, cyanobactérie réputée toxique pour certaines composantes de la biocénose du milieu ambiant (Oudra et al. 1997) et particulièrement pour certaines bactéries pathogènes (Oufdou et al. 1998), nous a incité à tester l'action d'un ajout supplémentaire de $\mathrm{NH}_{4} \mathrm{Cl}$ dans ce microcosme. Ce test a permis effectivement de confirmer l'absence d'effet toxique sur les nitrifiants (Fig. 6). Les concentrations de $\mathrm{NO}_{2}$ et de $\mathrm{NO}_{3}$ ont montré une élévation importante à partir du 6 ème jour atteignant, respectivement en fin d'expérience, des valeurs de 10.9 et $10.4 \mathrm{mg} \mathrm{N}^{-1}$. 

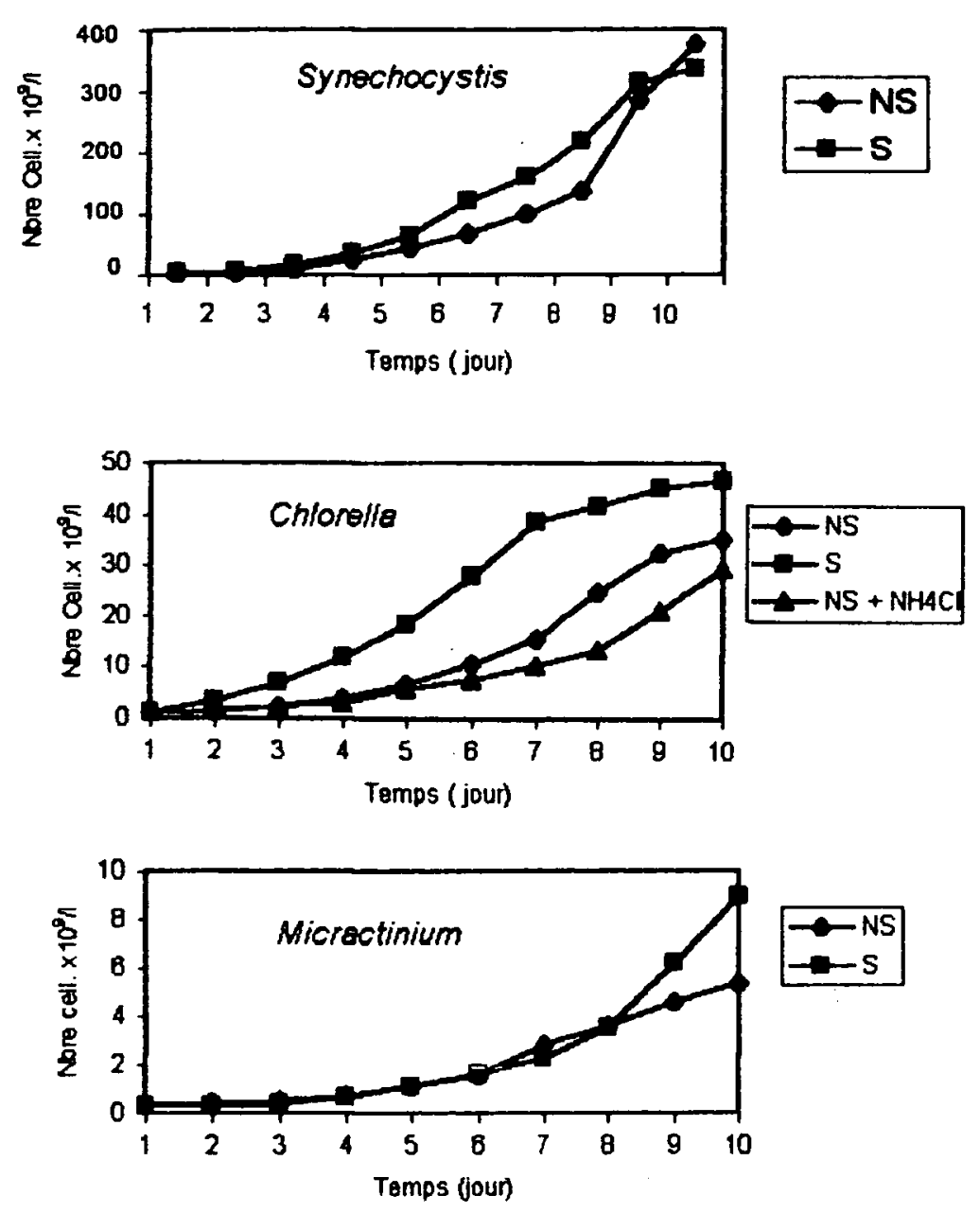

Fig. 7. Croissance de Synechocystis, de Chlorella et de Micractinium en milieu stérile (S) et non stérile (NS). $\mathrm{NS}+\mathrm{NH}_{4} \mathrm{CL}=\mathrm{En}-$ richissement en $\mathrm{NH}_{4} \mathrm{CL}$.

Fig. 7. Algal growth behaviour of Synechocystis, Chlorella and Micractinium incubated under sterilized (S), non-sterilized (NS) conditions. $\mathrm{NS}+\mathrm{NH}_{4} \mathrm{CL}=$ Enrichissement by $\mathrm{NH}_{4} \mathrm{CL}$.

\subsection{Croissance algale}

L'évolution de la densité des espèces d'algues montre une phase de latence et une phase exponentielle ; dans le cas de cette expérience qui s'arrête avec l'épuisement des nutriments azotés, la phase de déclin n'est pas atteinte. La densité des espèces algales passe, quel que soit le milieu, de $3.11 \times 10^{9}$ Cellules/L à $378 \times 10^{9}$ Cell. /L pour Synechocystis, de $1.24 \times 10^{9}$ Cell. /L à $46.65 \times 10^{9} \mathrm{Cell}$. /L pour Chlorella et de $0.4 \times 10^{9}$ à $9 \times 10^{9}$ Cell. /L pour Micractinium (Fig. 7). Pour des densités cellulaires d'ensemencement sensiblement équivalentes, les milieux stériles ont une production relativement supérieure aux milieux non stériles, bien que les taux de croissance maximale des différentes espèces n'aient pas trop varié entre les deux types de milieux de culture (Tableau 2 ). Le taux le plus élevé est constaté chez Synechocystis. En présence de Chlorella, l'enrichissement avec $\mathrm{NH}_{4} \mathrm{Cl}$ au 5 ème
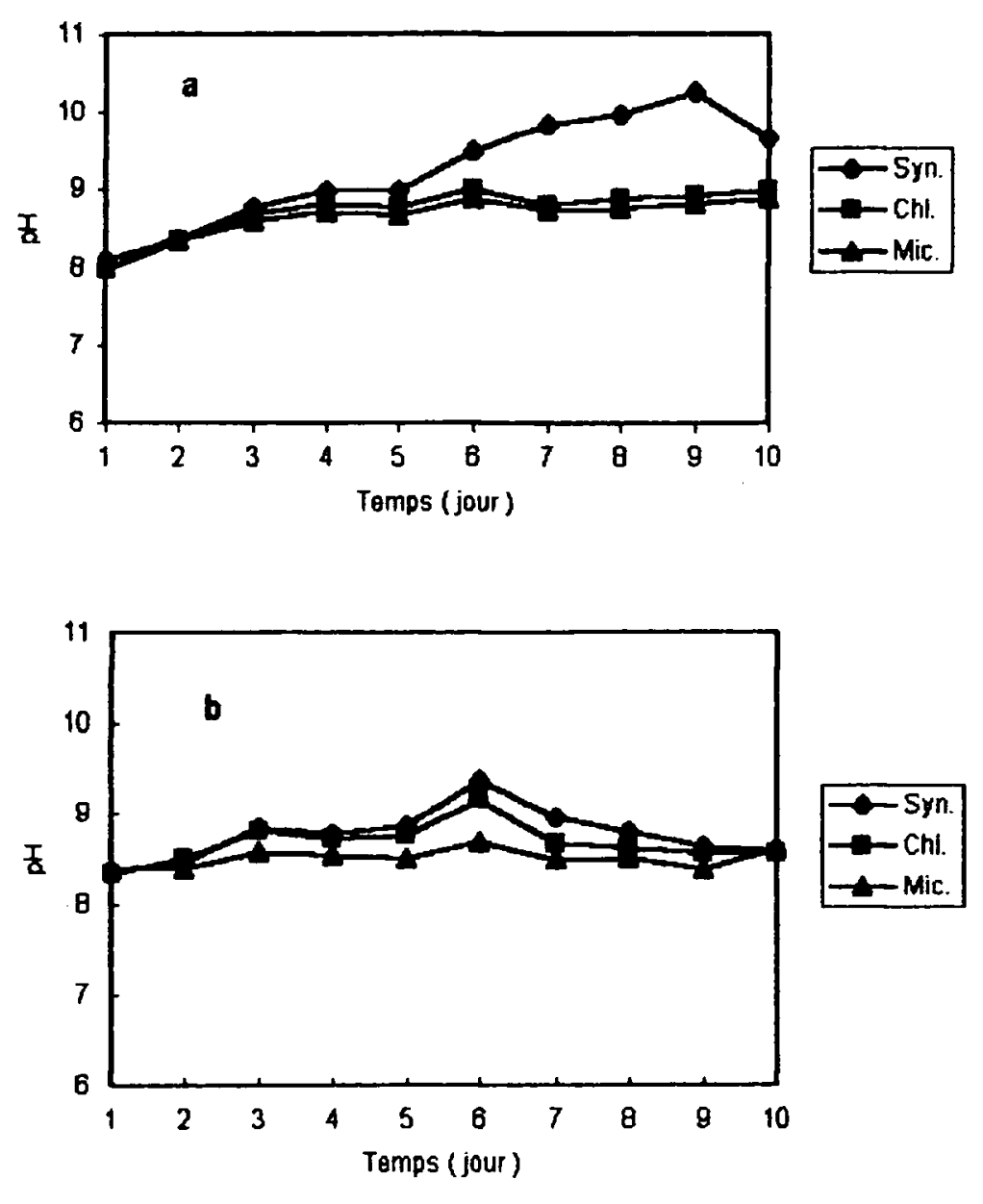

Fig. 8. Evolution temporelle du pH en présence de Synechocystis (Syn.), de Chlorella (Chl.) et de Micractinium (Mic.) en milieu non stérile (a) et stérile (b).

Fig. 8. Temporal changes in $\mathrm{pH}$ in non-sterilized (a) and sterilized (b) microcosms in the presence of Synechocystis (Syn.), Chlorella (Chl.) and Micractinium (Mic.).

jour n'a pas provoqué d'augmentation de la biomasse mais, au contraire, sa diminution accompagnée d'une baisse du taux de croissance. En nombre de cellules $\Lambda$, Synechocystis est de loin l'algue la plus productive suivie de Chlorella. Micractinium s'est révélée la moins productive. Cette productivité est d'ailleurs traduite dans les milieux réactionnels par l'élévation du $\mathrm{pH}$ (Fig. 8 ). En présence de Synechocystis, les valeurs du $\mathrm{pH}$, sont les plus élevées. En milieu non stérile, les valeurs du $\mathrm{pH}$ en présence de Chlorella et de Micractinium sont identiques alors qu'en milieu stérile les valeurs en présence de Chlorella sont plus élevées que celles en présence de Micractinium.

\subsection{Evolution des bactéries hétérotrophes aérobies}

Les bactéries hétérotrophes aérobies suivent une évolution plus au moins variable au cours de l'expérimentation (Tableau 3 ). L'augmentation du nombre de bactéries exprimé en log (U.F.C $/ \mathrm{ml}$ ) est importante en 
présence de Chlorella; ce nombre a varié de 7.04 à 7.74 , correspondant à une élévation de 5 fois au cours de 6 jours. Durant cette même période, ces bactéries ont augmenté de 1,5 fois en présence de Synechocystis et de 1,8 fois en présence de Micractinium.

\section{Discussion}

L'analyse des figures 1 à 5 montre que l'élimination de $\mathrm{NH}_{4}$ en milieux non stériles se fait par plusieurs mécanismes à savoir : l'assimilation algale, le stripping de $\mathrm{NH}_{3}$ et la nitrification et uniquement par les deux premiers mécanismes en milieux stériles. Dans les milieux non stériles et pendant les premiers jours, il apparaît que l'assimilation algale est le processus initialement le plus important qui s'associe à la nitrification et au stripping à partir du 5 ème jour. Sur la base des valeurs mentionnées dans le Tableau 4, on estime dans le milieu non stérile, par rapport à l'azote total Kjeldahl
(NTK), l'assimilation par les algues de $\mathrm{NH}_{4}$ à $23.3 \%$ pour Synechocystis, $14.7 \%$ pour Chlorella et $0.33 \%$ pour Micractinium; la nitrification est estimée à $1 \%$ en présence de Synechocystis, $8.5 \%$ en présence de Chlorella et à $11.4 \%$ en présence de Micractinium.

Par rapport à la concentration de $\mathrm{NH}_{4}$ initiale, le pourcentage des différentes voies dépasse $100 \%$, suggérant ainsi un apport des ions ammoniums par ammonification, mais aussi la possibilité d'une assimilation de l'azote organique directement par les microalgues surtout en milieux stériles. Le taux de nitrification est certainement sous- estimé, à cause de la dénitrification et de l'assimilation algale des nitrites et des nitrates. En effet, les algues assimilent les formes oxydées de l'azote, après épuisement de l'ammonium dans le milieu. Plusieurs auteurs rapportent que les algues, en présence des différentes formes de l'azote minéral, préfèrent $\mathrm{NH}_{4}$ qui est la forme azotée la moins exigeante sur le plan énergétique. Cependant, Robert \&

Tableau 2. Taux de croissance maximum $\left(\mathrm{J}^{-1}\right)$ des espèces algales.

Table 2. Algal maximum growth rate $\left(\right.$ day $\left.^{-1}\right)$.

\begin{tabular}{ccccccc}
\hline \multicolumn{2}{c}{ Synechocystis } & \multicolumn{3}{c}{ Chlorella } & \multicolumn{2}{c}{ Micractinium } \\
\hline S & NS & S & NS & NS* & S & NS \\
0.93 & 0.96 & 0.80 & 0.70 & 0.60 & 0.58 & 0.61 \\
\hline
\end{tabular}

$\mathrm{S}:$ Stérile ; NS : Non stérile ; $\mathrm{NS}^{*}$ : Non stérile et enrichi en $\mathrm{NH}_{4} \mathrm{CI}$

Tableau 3. Evolution temporelle des bactéries hétérotrophes aérobies (en $\log$ u. f. c. / ml) dans l'eau usée non stérile en présence des microalgues : Synechocystis, Chlorella et Micractinium.

Table 3. Growth behaviour of heterotrophic bacteria (log. u. f. c. / ml) incubated in no sterilized microcosm in the presence of microalgae : Synechocystis, Chlorella and Micractinium.

\begin{tabular}{cccc}
\hline & Synechocystis & Chlorella & Micractinium \\
\hline $\mathrm{t}=0$ & 7.56 & 7.04 & 7.39 \\
$\mathrm{t}=3$ & 7.61 & 7.53 & 7.51 \\
$\mathrm{t}=6$ & 7.73 & 7.74 & 7.64 \\
$\mathrm{t}=9$ & 7.55 & 7.62 & 7.44 \\
\hline
\end{tabular}

$t=$ temps en jour

Tableau 4. Evaluation des pourcentages d'assimilation des algues, de la nitrification et du stripping de $\mathrm{NH}_{4}{ }^{+}$en présence de Synechocystis, de Chlorella et de Micractinium dans l'eau usée non stérile.

Table 4. Evaluation of $\mathrm{NH}_{4}{ }^{+}$removal rate under non-sterilised condition by algal uptake, nitrification and $\mathrm{NH}_{3}$ stripping processus.

\begin{tabular}{cccc}
\hline & Synechocystis & Chlorella & Micractinium \\
\hline Nitrification & 1.00 & 8.50 & 11.40 \\
N algal & 23.30 & 14.70 & 0.33 \\
Stripping NH3 & 0.92 & 0.67 & 0.43 \\
\hline
\end{tabular}


Maestrini (1986) notent une assimilation concomitante de $\mathrm{NH}_{4}$ et de $\mathrm{NO}_{3}$ en deçà d'un seuil de $\mathrm{NH}_{4}$, seuil qui est fonction des espèces phytoplanctoniques. Dans cette préférence, l'inhibition de la nitrate réductase par $\mathrm{NH}_{4}$ est aussi incriminée Syrett 1981). Torre et al. (1992) ont rapporté que le déroulement de la dénitrification est possible, même dans un milieu oxygéné. En présence de Synechocystis, l'absence quasi totale de la nitrification peut être expliqué, à première vue, par l'effet toxique probable de cette cyanobactérie sur les bactéries nitrifiantes. En effet, Synechocystis peut avoir un effet toxique sur le zooplancton (Oudra et al. 1997) et sur certaines bactéries pathogènes (Oufdou et al. 1998). Cependant, les ajouts de $\mathrm{NH}_{4} \mathrm{Cl}$ que nous avons testé sur le microcosme en présence de cette microalgue (Fig. 6) montrent d'une manière claire que l'effet toxique sur les bactéries nitrifiantes est à écarter. De la comparaison de ces pourcentages, il ressort que les taux d'assimilation de $\mathrm{NH}_{4}$ sont inversement proportionnels à la nitrification. Ceci laisse présager que les algues et les bactéries nitrifiantes entrent en compétition nutritionnelle vis-à-vis de $\mathrm{NH}_{4}$. Cette Hypothèse est corroborée par la légère différence de comportement des espèces algales entre les milieux stérile et non stérile. Le milieu stérile dans lequel les bactéries nitrifiantes sont absentes d'où absence de compétition bactéries - algues, est plus productif que le milieu non stérile. Dans une revue bibliographique sur les interactions algues - bactéries, Cole (1982) évoque une compétition pour le nutriment limitant dans le milieu comme une action inhibitrice des bactéries sur la croissance algale. Rhee (1972) a également constaté dans un microcosme en laboratoire, une compétition vis-à-vis du phosphore entre Scenedesmus et Pseudomonas, ce qui entraîne une diminution du phosphore intracellulaire chez l'algue testée. Cette compétition nutritionnelle est remportée par l'organisme le plus performant sur le plan métabolique vis-à-vis des nitrifiants. Ainsi, l'ordre d'activité métabolique suivant peut être dressé : Synechocystis, Chlorella, Micractinium. Le rapport biosurface / biovolume peut être in- voqué comme facteur explicatif dans ce classement (Reynolds 1984). Dans le même ordre d'idées, la taille des cellules peut expliquer la prédominance de telle ou telle espèce d'algue sur les nitrifiants. Dauta \& Feuillade (1995) rapportent que la vitesse d'absorption d'un élément nutritif diminue lorsque le diamètre des cellules augmente. De par sa petite taille $(1 \mu \mathrm{m}$ de diamètre), Synechocystis se trouve avantagée dans cette compétition algues - bactéries, par rapport à Chlorella $(3 \mu \mathrm{m})$ et à Micractinium $(8 \mu \mathrm{m})$. L'importance des stocks intracellulaires n'est pas à écarter ; selon Dauta (1982), ce stock augmente, d'une manière générale, avec le biovolume des cellules et varie en fonction des espèces, de la taille des cellules et du nutriment (Dauta \& Feuillade 1995). Cette activité métabolique des microalgues de petite taille est soulignée par les valeurs de la biomasse ( en Chlorophylle a ) atteintes respectivement par Synechocystis, (5032.7 $\left.\mu \mathrm{g} . \mathrm{l}^{-1}\right)$, Chlorella (3075.6 $\mu \mathrm{g} .1^{-1}$ ) et Micractinium $\left(73.6 \mu \mathrm{g} . \mathrm{l}^{-1}\right)$ (Tableau 5). Les valeurs du $\mathrm{pH}$ enregistrées selon les différentes microalgues (Fig. 8), traduisent l'activité métabolique de chacune des ces espèces. En présence de Synechocystis, espèce la plus productive, le $\mathrm{pH}$ est plus élevé qu'en présence de Chlorella et de Micractinium.

La chute des $\mathrm{NH}_{4}$, constatée entre le $5^{\text {ème }}$ et le 6 ème jour, est principalement due à l'activité algale, dans le cas de Synechocystis et à la nitrification dans le cas de Micractinium et aux deux processus, en présence de Chlorella. Le taux de nitrification, au 6 ème jour, basé sur la somme de $\mathrm{NO}_{2}$ et de $\mathrm{NO}_{3}$ par rapport à la concentration de $\mathrm{NH}_{4}$ au 4 ème jour, juste après cette chute, est de $11.6 \%$ en présence de Synechocystis, $21.7 \%$ en présence de Chlorella et de $88.1 \%$ en présence de Micractinium. Dans des études antérieures avec Scenedesmus, De La Noue et al. (1989) suggèrent que l'épuisement presque total de $\mathrm{NH}_{4}$ dans un milieu de culture constitué d'eau usée domestique est dû principalement à la consommation algale et n'évoque ni la nitrification ni le stripping. Cette situation est comparable aux résultats obtenus avec Synechocystis. Przytocka-Jusiak et al. (1984) ont montré que l'incapacité

Tableau 5. Teneurs en chlorophylle a ( $\left.\mu \mathrm{g} \cdot \mathrm{l}^{-1}\right)$ des microalgues, Synechocystis, Chlorella et Micractinium.

Table 5. Chlorophyll a content ( $\mu$ g.1-1) of microalgae species, Synechocystis, Chlorella and Micractinium.

\begin{tabular}{cccc}
\hline & Synechocystis & Chlorella & Micractinium \\
\hline T1 & 799.5 & 108.5 & 5.6 \\
T5 & 2798.2 & 594.1 & 14.8 \\
T10 & 5032.7 & 3075.6 & 73.6 \\
\hline
\end{tabular}

$T=$ temps en jour 
des algues à éliminer la totalité de $\mathrm{NH}_{4}$ des eaux usées, favorise la croissance des bactéries nitrifiantes. En effet, l'enrichissement que nous avons effectué en présence de Chlorella n'a pas provoqué d'augmentation de la biomasse algale, mais au contraire une diminution. Cependant, l'effet le plus remarquable est constaté sur la nitrification (Figs. 5 et 7 ). La diminution de la densité cellulaire algale causée par cet enrichissement est certainement attribué à $\mathrm{NH}_{3}$ ( forme non ionique ) qui est considéré comme toxique pour le phytoplancton, en ce sens qu'il induit une inhibition de l'activité photosynthétique (Abeliovich \& Azov 1976, Pearson et al. 1987, Konig et al. 1987). L'apparition de $\mathrm{NH}_{3}$ a été effectivement révélée à partir du 3 ème jour avec des pH dépassant 8,5 (Figs. 2 et 8 ). Cette forme de l'azote constitue, par l'intermédiaire de son stripping, la voie chimique de l'élimination de $\mathrm{NH}_{4}$. En effet, les pertes engendrées par ce processus sont respectivement estimées à 7.7, 5.5 et $3.8 \%$ de l'ammonium initial, en présence de Synechocystis, de Chlorella et de Micractinium. Picard et al. (1979) ont montré, avec une augmentation du $\mathrm{pH}$ par $\mathrm{NaOH}$, que les pertes de $\mathrm{NH}_{3}$ restent relativement faibles à des $\mathrm{pH}$ inférieurs à 10 et d'autant plus que le contact air - eau est restreint. Vu que les différentes voies citées dépassent $100 \%$ d'élimination de $\mathrm{NH}_{4}$ initial, il est vraisemblable que cette forme de l'azote n'est pas affectée par les bactéries hétérotrophes aérobies, compte tenu de l'abondance de l'azote organique dissous dans le milieu réactionnel. Ceci est d'autant plus probable que la méthode d'abondance bactérienne utilisée sous-estime cette population. En effet, il existe des bactéries qualifiées de «bactéries viables non cultivables» qui sont métaboliquement actives mais incapables de croître sur boite de pétri (Servais et al. 1992). Nous pouvons donc avancer sans trop de risques que par cette méthode nos résultats ne peuvent qu'être confortés. Les travaux de Downing et al., cités par Wood et al. (1981) ont révélé que dans les eaux usées la concentration de l'ammonium n'est pas affectée par l'activité des bactéries hétérotrophes. L'azote organique dissous dans les eaux du lagunage, estimé en moyenne à $34 \mathrm{mg} \mathrm{N} / \mathrm{l}$ ( Bouarab et al. 1994), est probablement riche en acides aminés à faible poids moléculaire (D.F.A.A.) qui sont utilisés préférentiellement par les bactéries hétérotrophes aérobies (Coveney et al. 1977, Kirchman 1994, Kirchman et al. 1994). Dauta \& Feuillade (1995) rapportent que le phytoplancton excrète abondamment du glycolate, de la glycine et de la serine (métabolites du cycle oxydatif de Tolbert) sous de fortes teneurs en oxygène dissous, conditions qui peuvent se rencontrer dans notre milieu réactionnel

\section{Conclusion}

L'étude des voies d'élimination de $\mathrm{NH}_{4}$ des eaux usées en présence de trois microalgues de différentes tailles a été effectuée sur milieux stérile et non' stérile. Cette étude montre l'existence de trois mécanismes d'élimination de $\mathrm{NH}_{4}$, à savoir l'assimilation algale, le stripping de $\mathrm{NH}_{3}$ et la nitrification ; ce dernier mécanisme est absent dans le milieu stérile. Les taux des différents mécanismes sont respectivement de 23.3, 0.92 et $1 \%$ en présence de Synechocystis, $14.7,0.67$ et $8.5 \%$ en présence de Chlorella et enfin de $0.33,0.43$ et $11.4 \%$ en présence de Micractinium dans le milieu non stérile. La comparaison de ces pourcentages montre que l'assimilation et le stripping sont inversement proportionnels à la nitrification, suggérant ainsi une compétition entre les algues et les bactéries nitrifiantes. La taille des cellules algales - ou le rapport biosurface / biovolume - est invoquée dans cette compétition, les petites cellules sont plus performantes que les grosses cellules vis-à-vis des bactéries nitrifiantes. Après épuisement de $\mathrm{NH}_{4}$ dans le milieu réactionnel, en présence de Chlorella, son enrichissement en cette forme d'azote, entraine non pas une augmentation de la biomasse algale, mais plutôt une activation de la nitrification; ceci laisse présager à l'incapacité des algues à éliminer la totalité de $\mathrm{NH}_{4}$ du milieu. En présence de Synechocystis, le faible pourcentage de la nitrification n'est pas due à un effet toxique de celle-ci sur les bactéries nitrifiantes.

A la lumière des pourcentages évalués pour chaque voie d'élimination de $\mathrm{NH}_{4}$, on peut conclure que les bactéries hétérotrophes aérobies n'interviennent pas dans cette élimination, probablement à cause de leur préférence pour l'azote organique présent dans le milieu ou excrété par les algues.

\section{Travaux cités}

Abeliovich A. \& Azov Y. 1976.- Toxicity of ammonia to algae in sewage oxidation ponds. Appl. envir. Microbiol., 31 : 801-806.

Abeliovich A. 1992.- Transformations of ammonia and the environmemtal impact of nitrifyng bacteria. Biodegradation, 3 :255264.

Bouarab L., Ouazzani N., Ould El Mehdi M. ,Loudiki M.\& Picot B. 1994 -- -Evolution des formes d'azote dans la station expérimentale de lagunage naturel de Marrakech. IVème Conférence Internationale des Limnologues d'Expression Française (CILEF 4), Bien gérer nos ressources pour mieux protéger notre environnement, Marrakech (Maroc) 25-28 Avril.

Caperon J. \& Meyer J. 1972.- Nitrogen limited growth of marine phytoplankton changes in population characteristics with steadystate growth rate. Deep-sea Research, $19: 601-618$.

Cole J.J. 1982. - Interactions between bacteria and algae in aquatic ecosystems. Ann. Rev. Ecol. Syst., 13 : 291-314.

Coveney M.F., Cronberg G., Enell M., Larsson K. \& Olofsson L. 1977.- Phytoplankton, zooplankton and bacteria- standing crop and production relationships in a eutrophic lake. Oikos, 29, (1) :5-21. 
Dauta A. \& Feuillade J. 1995.- Croissance et dynamique des populations algales. in Limnologie générale, Pourriot R. \& Meybeck M.,(Eds.) Masson, Paris : 328-350.

Dauta A. 1982.-Conditions de développement du phytoplancton. Etude comparative du comportement de huit espèces en culture. II - Rôle des nutriments : assimilation et stockage intracellulaire. Annls. Limnol.,18 (3) : 263-292.

Dauta A., Brunel L. \& Guerri M. 1982.- Détermination expérimentale des paramètres liés à l'assimilation de l'azote et du phosphore par Scenedesmus crassus. Annls. Limnol., 18 (1) : 33-40.

De la Noue J., Van coillie R., Brunel C. \& Pouliot Y. 1989.- Traitement des eaux usées par culture de microalgues : Influence de la composition du milieu sur la croissance de Scenedesmus sp. Annls. Limnol. 25 (3) : 197-203.

Dunstan W.H. \& Menzel D.W. 1971.- Continuous cultures of natural populations of phytoplankton in dilute treated sewage effluent. Limnol. \& Oceanog., $16(4)$ : 623-632.

Ellis K.V. 1983.- Stabilization ponds : design and operation. Critical Reviews in Environnmental Control, 13 (2) : 69-102.

Humenik F.J. \& Hanna G.P. 1971.- Algal-Bacterial symbiosis for removal and conservation of wastewater nutrients. Journal WPCF, 43 (4) : 580-594.

Kirchman D.L. 1994. - The uptake of inorganic nutrients by heterotrophic bacteria. Microb. Ecol., $28: 255-271$.

Kirchman D.L., Ducklow H.W., McCarthy J.J. \& Garsides C. 1994.- Biomass and nitrogen uptake by heterotrophic bacteria during the spring phytoplankton bloom in the North Atlantic Ocean. Deep-Sea Research, 41 (5/6) : 879-895.

Konig A., Pearson H.W. \& Silva S.A. 1987.- Ammonia toxicity to algal growth in waste stabilization ponds. Wat. Sci. Tech., 19 (12) : 115-122.

Lavoie A. \& De la Noue J. 1985.- Hyperconcentrated cultures of Scenedesmus obliquus. A new approch for wastewater biological tertiary treatment. Wat. Res., 19 (11) : $1437-1422$.

Marker A.F.H., Nush E.A.\& Riemann B. 1980.--The measurements of photosynthetic pigments in freshwater and standarization of methods : conclusions and considerations of the workshop. Arch. Hydrobiol./Beih. Ergebn. Limnol., 14 : 91-106.

Middlebrooks E.J. \& Panno A. 1983.- Nitrogen removal in aerated lagoons. Wat. Res., 17 (10) : 1369-1378.

Mujeriego R., Sala L. \& Turet J. 1993.- Nutrients losses in two landscale ponds used for Golf course irrigation. 2nd international specialist conference, International Association on Water Quality ( $I A W Q$ ), waste stabilization ponds \& the reuse of pond effluents, Berckeley (California USA), 30th November-3rd December.

Neel J.K, McDermott J.H. \& Monday C.A. 1961.- Experimental lagooning of raw sewage at Fayette, Missouri. Journal WPCF, 33 (6) : 603-641.

Oufdou Kh., Mezrioui N., Barakate M. \& Ouhdouch I. 1998.— Etude expérimentale de l'effet de Synechocystis sp.(picocyanobactérie) sur le comportement de certaines bactéries d'intérêt sanitaire. Annls Limnol., 34 (3) : 259-268.
Oudra B., Piedade B.; Vascancelos V. \& Loudiki M. 1997.- Toxic effects of Cyanobacterium Synechocystis parvula Perfliew on Daphnia magna Straus. VIIIth international conference on harmful algae, Vigo ( Spain), 25-29 June.

Pearson H., W. Mara D.D., Mills S.W. \& Smallman D.J. 1987.Factors determining algal populations in waste stabilization ponds and the influence of algae on pond performance. Wat. Sci. Tech., 19 (12) : 131-140.

Picard G.A., De la Noue J., Piette J.P. \& Kirouac C. 1979.- Utilisation de l'algue Oocystis sp. pour le traitement tertiaire des eaux usées - 1- Culture en vrac de cellules préalablement conditionnées en cyclostat. Wat. Res., $13: 1203-1212$.

Przytocka-Jusiak M., Blaszczyk M., Kosinka E. \& Bisz-Konarzewska A. 1984.- Removal of nitrogen from industrial wastewaters with the use of algal rotating disks and denitrification packed bed reactor. Wat. Res., 18 (9) : 1077-1082.

Reynolds. C.S. 1984. - The ecology of fresh water phytoplankton. University Press, Cambridge : 374 p.

Rhee G.Y. 1978. - Effects of $\mathrm{N}: \mathrm{P}$ atomic ratios and nitrate limitation on algal growth, cell composition, and nitrate uptake. Limnol. Oceanogr., 23 (1) 10-23.

Robert J. M. \& Maestrini S.Y. 1986.- Absorptions simultanées des ions $\mathrm{NO}_{3}{ }^{-}$et $\mathrm{NH}_{4}{ }^{+}$par trois diatomées de claires à huîtres, en culture axénique. Phycologia, 25 (2) :125-159.

Santos H.C.R. \& Olveira F.S. 1987.- Nitrogen transformations and removal in waste stabilization ponds in Portugal : saesonal variations. Wat. Sci. Tech., 19 (12) : 123-130.

Servais P., Laurent P. \& Randon G. 1992.- Mesure de la biomasse et de l'activité bactérienne dans l'eau de distribution. Revue des Sciences de l'Eau, $5:$ 473-488.

Silva S.A., Oleveira R., Soares J. \& Mara D.D. 1993. - Nitrogen removal in pond systems with different configurations and geometries. 2nd international specialist conference, International Association on Water Quality (IAWQ), Waste stabilization ponds \& the reuse of pond effluents, Berckeley (California USA), 30th November-3rd December.

Somiya I. \& Fujii S. 1984.- Material balances of organics and nutrients in an oxidation pond. Wat. Res., 18 (3) : 325-333.

Syrett P.J. 1981.- Nitrogen metabolism of microalgae. Physiological Bases of phytoplankton Ecology. T. Platt ( Ed.), Can. Bull. Fish. Aquat. Sci., $210: 182-204$.

Torre M., Rebillard J.P., Ayphassorho H., Labroue L. \& Helmer C. 1992. - Etude expérimentale de la dénitrification in situ en eaux courantes : application à la rivière Charente. Annls. Limnol., 28 (3) : 263-271.

Walker J. \& Leclerc H. 1973.- Traitement expérimental d'épuration d'une eau de surface par lagunage : aspects chimiques et microbiologiques. Wat. Res., 7 : 707-728.

Wood L.B., Hurley B.J.E. \& Mathews P.J. 1981.- Some observations on the biochemistry and inhibition of nitrification. Wat. Res., 15 : 543-551.

Wrigley T.J. \& Toerien D. F. 1990._ Limnological aspects of small sewage ponds. Wat. Res., $24: 83-90$. 